\title{
Disease stratification in idiopathic pulmonary fibrosis: the dawn of a new era?
}

\author{
Toby M. Maher ${ }^{1,2,3}$
}

Affiliations: ${ }^{1}$ NIHR Biological Research Unit, Royal Brompton Hospital, London, ${ }^{2}$ Faculty of Medicine, National Heart and Lung Institute, Imperial College London, London, and ${ }^{3}$ Centre for Inflammation and Tissue Repair, University College London, Rayne Institute, London, UK.

Correspondence: T.M. Maher, NIHR Biological Research Unit, Royal Brompton Hospital, Sydney Street, London, SW3 6NP, UK. E-mail: t.maherarbht.nhs.uk

0

@ERSpublications

Measurement of serum LOXL2 brings the prospect of stratified medicine one step closer for patients with IPF http://ow.ly/tv6VQ

Research into idiopathic pulmonary fibrosis (IPF) has been rapidly gathering pace over the past few years. This has been driven by a number of important factors, including the establishment of both clinical and basic science research networks, growing involvement of the pharmaceutical industry, and an exponential rise in the recruitment of patients into clinical trials $[1,2]$. These changes have resulted in an improved understanding of disease pathogenesis and have culminated in the licensing, in Europe, of the first IPFspecific therapy, pirfenidone. However, the dismal prognosis for sufferers of the disease remains, as yet, unchanged. Why is this? And, more importantly, what can be done to improve survival for individuals with IPF?

IPF is a challenging disease. At diagnosis the condition is typically characterised by established fibrosis with architectural destruction of the lung and loss of gas exchange surface area. A growing body of evidence points to IPF arising as the consequence of an aberrant wound healing response occurring after repetitive alveolar injury in genetically susceptible individuals. As such, multiple cascades, all of which play a role in normal wound healing, are involved in the progressive fibro-proliferation that characterises $\operatorname{IPF}[3,4]$. These abnormalities in wound healing-related mechanisms result in a myriad of growth factors, cytokines, chemokines and lipid mediators being differentially regulated in the lungs of patients with IPF [4]. This abnormal interplay of cell-cell interactions and signalling cascades is redolent of the abnormalities observed in cancer [5]. Therefore, it is perhaps no coincidence that diagnostic and therapeutic developments in the field of oncology provide a potential road map for improving outcomes in IPF.

Until relatively recently, drug discovery across all branches of medicine, but particularly oncology, was driven by the search for golden bullets; medications which, at a single stroke, would cure all sufferers with a given disease. As understanding of the pathobiology of disease has improved it has become increasingly clear that such a simplistic, one size fits all approach is destined to failure. Even in single gene disorders such as cystic fibrosis, it is now evident that treatment responses vary between individuals. Ivacafcor, a novel peptide capable of restoring cystic fibrosis transmembrane regulator function, results in striking improvements in lung function and symptom scores, but only in the $2-5 \%$ of cystic fibrosis patients carrying the G551D mutation [6]. Given that such complexity exists in a well-understood monogenetic disease, what can we hope to learn when it comes to diseases characterised by multiple pathway abnormalities such as cancer and IPF?

Received: Dec 312013 | Accepted: Jan 042014

Conflict of interest: Disclosures can be found alongside the online version of this article at www.erj.ersjournals.com 
The recognition that diseases with a single distinct clinical phenotype can arise through multiple mechanisms has given rise to the development of stratified medicine [7]. At its core stratified medicine relies on the use of modern molecular tools, such as genomics, transcriptomics and proteomics (to name but a few of the 'omics), to identify clusters of patients with a given disease who share a specific molecular phenotype. In oncology, the advent of molecular phenotyping has, for many cancers, enabled better prognostication, which in turn has enable oncologists to utilise existing treatments according to likelihood of disease progression [8]. Perhaps more importantly, however, molecular phenotyping has enabled the novel therapies to be targeted at individuals most likely to respond. A prime example of this is gleevec, a Bcr-Abl tyrosine kinase inhibitor, which has transformed outcomes in individuals with Philadelphia chromosome-positive chronic myeloid leukaemia [9]. Such an approach has the benefit of limiting exposure to costly and potentially toxic treatments to only those individuals likely to derive a benefit. Stratified treatment, whilst most advanced in oncology, has begun to emerge in other disease areas. In asthma, the novel anti-interleukin-13 monoclonal antibody, lebrikizumab, has been shown to improve airflow obstruction in individuals with elevated levels of the serum protein periostin, but not those with normal levels [10]. Whilst requiring further validation, this observation suggests that biomarker-driven therapy may become a reality in respiratory medicine in the near future [11]. For a disease as complex as IPF, stratification intuitively makes sense but what should come first, the treatment or the biomarker?

At the heart of stratified medicine sit biomarkers. These are characteristics that are objectively measured and evaluated as an indicator of normal biological processes, pathogenic processes or pharmacological responses to therapeutic interventions [12]. Biomarkers, whilst most commonly thought of as serum proteins, can be based on modalities as diverse as imaging or measurements of pulmonary physiology [13, 14]. Biomarkers can be developed for a range of purposes, including diagnosis, predicting future disease progression, determining response to therapy, or detecting the development of disease specific complications, e.g. pulmonary hypertension. In the context of stratified medicine a biomarker would be expected to define a group of patients likely to respond to a given therapy [7]. A number of putative serum biomarkers have been identified in IPF. The best described of these are matrix metalloproteinase (MMP)-7, chemokine (C-C motif) ligand (CCL)-18 and KL-6 [15-17]. However, in each case these markers have typically been identified in pre-existing sample collections from single centres and with blood drawn at a single time point. Furthermore, whilst these markers may yet prove to be effective for identifying individuals with progressive disease, they do not map biological pathways for which potential IPF therapies exist [18]. For this reason the manuscript by CHIEN et al. [19] in the current issue of the European Respiratory Journal represents an exciting development for IPF.

The lysyl oxidase-like (LOXL) proteins are a group of five enzymes that facilitate the cross-linking of type 1 collagen molecules and which, therefore, are important in extracellular matrix deposition [20]. One of these proteins, LOXL2, which is synthesised and secreted by fibroblasts, has been shown to be particularly important in driving collagen accumulation, deposition and stiffening whilst also promoting cell proliferation [21]. In the murine bleomycin-induced lung fibrosis model, the anti-LOXL2 monoclonal antibody, simtuzumab (GS-6624 and AB0024; Gilead Science Inc., Seattle, WA, USA), blocks fibrosis and appears to do so by reducing production of fibrillar collagen, preventing fibroblast activation and inhibiting the release of inflammatory cytokines including transforming growth factor- $\beta 1$ [21]. Simtuzumab is currently being studied in a phase $2 \mathrm{~b}$ trial as a potential treatment for IPF (www.ClinicalTrials.com, identifier NCT01769196).

LOXL2 has previously been shown to be detectable in serum [22]. CHIEN et al. [19] have utilised two existing IPF cohorts (drawn from the ARTEMIS-IPF study [23] and the GAP (Genomic and Proteomic Analysis of Disease Progression in Idiopathic Pulmonary Fibrosis) study) to explore the relationship between serum (s)LOXL2 levels and subsequent disease progression and mortality. Their data demonstrate that sLOXL2 is elevated in IPF patients compared to healthy controls and that levels weakly correlate with baseline disease severity (as measured by forced vital capacity and diffusing capacity of the lung for carbon monoxide) [19]. However, more importantly, in both cohorts elevated sLOXL2 correlated with an increased risk of disease progression (defined as a composite of mortality, respiratory hospitalisation or a categorical decline in lung function). In the GAP cohort, elevated sLOXL2 levels were also associated with increased mortality.

Taken at face value these results appear impressive. However, there are a number of key weaknesses with the study that need to be considered when interpreting the data. Only $14 \%(n=69)$ of the ARTEMIS-IPF participants had baseline serum samples available for inclusion in this study. Of these, two-thirds of subjects were in the active treatment arm and so received ambrisentan; something that may have had a negative effect on disease outcomes. Follow-up of the ARTEMIS-IPF cohort was, on average, limited to only 245 days and so there were relatively few disease progression events and only nine deaths. The GAP cohort was recruited from a large transplant centre in the USA and, as such, had relatively severe disease at 
enrolment. Approximately one-third of patients in the GAP study were missing baseline lung function data and so could not be included in the disease progression analysis. Furthermore, information on hospitalisation was not collected in the GAP study meaning that this was not included in the disease progression composite end-point, even for the two-thirds of subjects with baseline lung function data. Whilst the available follow-up was longer in the GAP cohort, $16 \%$ of subjects underwent transplant. For the purposes of the statistical analyses transplants were counted as death. This introduces a potential bias as transplant will inevitably bring forward an individual's "date of death". In part, because of these differences sensitivity analysis identified different thresholds for sLOXL2 levels as a predictor of disease progression in the two cohorts.

Whilst these major methodological weaknesses need to be borne in mind, the fact that serum LOXL2 predicted disease progression in two very different cohorts of IPF patients is reassuring. Taken together, the data presented by CHIEN et al. [19] suggest that sLOXL2, like MMP7, CCL-18 and KL-6, has potential as a biomarker to identify individuals with IPF at increased risk of disease progression. However, what makes these results altogether more exciting is the fact that LOXL2 is a potential therapeutic target in IPF. The finding that sLOXL2 is elevated in individuals with IPF when compared to controls reinforces the biological plausibility of this target. More importantly, sLOXL2 measurements may enable disease stratification and, thus, better targeting of anti-LOXL2 therapy.

As has been argued elsewhere, biomarker discovery and disease stratification in IPF requires well-designed, prospective, multicentre studies of appropriately phenotyped patients with collection of longitudinal outcome data [1]. Furthermore, to better understand the value of measuring serum proteins, the collection of biological samples should not just occur at baseline but needs to be repeated at multiple time points. It is only through such studies being conducted that it will be truly possible to validate the role of potential biomarkers. Despite its major limitations, the study by CHeIN et al. [19] should provide the necessary impetus for further research into sLOXL2 as a disease progression biomarker and stratification tool in IPF. If it succeeds in doing so, the study will represent the dawning of an exciting new era in clinical IPF research.

\section{References}

1 Maher TM. PROFILEing idiopathic pulmonary fibrosis: rethinking biomarker discovery. Eur Respir Rev 2013; 22: $148-152$.

2 Richeldi L, Collard HR, du Bois RM, et al. Mapping the future for pulmonary fibrosis: report from the 17th International Colloquium on Lung and Airway Fibrosis. Eur Respir J 2013; 42: 230-238.

3 Maher TM, Wells AU, Laurent GJ. Idiopathic pulmonary fibrosis: multiple causes and multiple mechanisms? Eur Respir J 2007; 30: 835-839.

4 Wuyts WA, Agostini C, Antoniou KM, et al. The pathogenesis of pulmonary fibrosis: a moving target. Eur Respir J 2013; 41: 1207-1218.

5 Vancheri C, du Bois RM. A progression-free end-point for idiopathic pulmonary fibrosis trials: lessons from cancer. Eur Respir J 2013; 41: 262-269.

6 Ramsey BW, Davies J, McElvaney NG, et al. A CFTR potentiator in patients with cystic fibrosis and the G551D mutation. N Engl J Med 2011; 365: 1663-1672.

7 Maher TM. Beyond the diagnosis of idiopathic pulmonary fibrosis; the growing role of systems biology and stratified medicine. Curr Opin Pulm Med 2013; 19: 460-465.

8 Gonzalez de Castro D, Clarke PA, Al-Lazikani B, et al. Personalized cancer medicine: molecular diagnostics, predictive biomarkers, and drug resistance. Clin Pharmacol Ther 2013; 93: 252-259.

9 Druker BJ, Talpaz M, Resta DJ, et al. Efficacy and safety of a specific inhibitor of the BCR-ABL tyrosine kinase in chronic myeloid leukemia. N Engl J Med 2001; 344: 1031-1037.

10 Corren J, Lemanske RF, Hanania NA, et al. Lebrikizumab treatment in adults with asthma. N Engl J Med 2011; 365: 1088-1098.

11 Wheelock CE, Goss VM, Balgoma D, et al. Application of 'omics technologies to biomarker discovery in inflammatory lung diseases. Eur Respir J 2013; 42: 802-825.

12 Jones PW, Agusti AG. Outcomes and markers in the assessment of chronic obstructive pulmonary disease. Eur Respir J 2006; 27: 822-832.

13 Mura M, Porretta MA, Bargagli E, et al. Predicting survival in newly diagnosed idiopathic pulmonary fibrosis: a 3-year prospective study. Eur Respir J 2012; 40: 101-109.

14 du Bois RM, Albera C, Bradford WZ, et al. 6-minute walk distance is an independent predictor of mortality in patients with idiopathic pulmonary fibrosis. Eur Respir J 2014; 43: 1421-1429.

15 Rosas IO, Richards TJ, Konishi K, et al. MMP1 and MMP7 as potential peripheral blood biomarkers in idiopathic pulmonary fibrosis. PLoS Med 2008; 5: e93.

16 Prasse A, Probst C, Bargagli E, et al. Serum CC-chemokine ligand 18 concentration predicts outcome in idiopathic pulmonary fibrosis. Am J Respir Crit Care Med 2009; 179: 717-723.

17 Yokoyama A, Kondo K, Nakajima M, et al. Prognostic value of circulating KL-6 in idiopathic pulmonary fibrosis. Respirology 2006; 11: 164-168.

18 Maher TM. Idiopathic pulmonary fibrosis: pathobiology of novel approaches to treatment. Clin Chest Med 2012; 33: 69-83.

19 Chien JW, Richards TJ, Gibson KF, et al. Serum lysyl oxidase-like 2 levels and idiopathic pulmonary fibrosis disease progression. Eur Respir J 2014; 43: 1430-1438.

20 Yamauchi M, Shiiba M. Lysine hydroxylation and cross-linking of collagen. Methods Mol Biol 2008; 446: 95-108. 
21 Barry-Hamilton V, Spangler R, Marshall D, et al. Allosteric inhibition of lysyl oxidase-like-2 impedes the development of a pathologic microenvironment. Nature Med 2010; 16: 1009-1017.

22 Makawita S, Dimitromanolakis A, Soosaipillai A, et al. Validation of four candidate pancreatic cancer serological biomarkers that improve the performance of CA19.9. BMC Cancer 2013; 13: 404.

23 Raghu G, Behr J, Brown KK, et al. Treatment of idiopathic pulmonary fibrosis with ambrisentan: a parallel, randomized trial. Ann Intern Med 2013; 158: 641-649. 